\title{
Automatic modular design of robot swarms using behavior trees as a control architecture
}

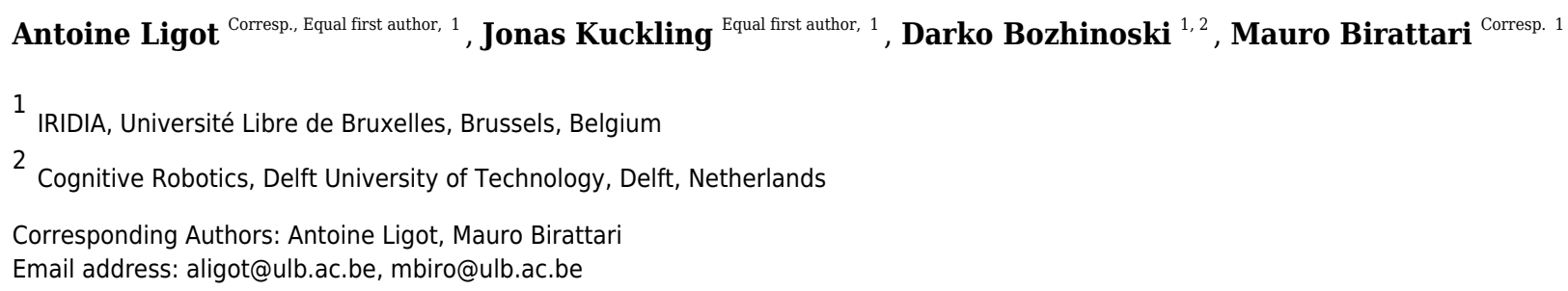

We investigate the possibilities, challenges, and limitations that arise from the use of behavior trees in the context of the automatic modular design of collective behaviors in swarm robotics. To do so, we introduce Maple, an automatic design method that combines predefined modules---low-level behaviors and conditions---into a behavior tree that encodes the individual behavior of each robot of the swarm. We present three empirical studies based on two missions: AGGREGATION and Foraging. To explore the strengths and weaknesses of adopting behavior trees as a control architecture, we compare Maple with Chocolate, a previously proposed automatic design method that uses probabilistic finite state machines instead. In the first study, we assess Maple's ability to produce control software that crosses the reality gap satisfactorily. In the second study, we investigate Maple's performance as a function of the design budget, that is, the maximum number of simulation runs that the design process is allowed to perform. In the third study, we explore a number of possible variants of Maple that differ in the constraints imposed on the structure of the behavior trees generated. The results of the three studies indicate that, in the context of swarm robotics, behavior trees might be appealing but in many settings do not produce better solutions than finite state machines. 


\title{
Automatic modular design of robot swarms using behavior trees as a control architecture
}

\author{
Antoine Ligot ${ }^{1}$, Jonas Kuckling ${ }^{1}$, Darko Bozhinoski ${ }^{1,2}$, and Mauro \\ Birattari $^{1}$ \\ ${ }^{1}$ IRIDIA, Université libre de Bruxelles \\ ${ }^{2}$ Cognitive Robotics, Delft University of Technology \\ Corresponding author: \\ Mauro Birattari \\ Email address: mbiro@ulb.ac.be
}

11 ABSTRACT

2 We investigate the possibilities, challenges, and limitations that arise from the use of behavior trees in the context of the automatic modular design of collective behaviors in swarm robotics. To do so, we introduce Maple, an automatic design method that combines predefined modules-low-level behaviors and conditions-into a behavior tree that encodes the individual behavior of each robot of the swarm. We present three empirical studies based on two missions: AGGREGATION and FORAGING. To explore the strengths and weaknesses of adopting behavior trees as a control architecture, we compare Maple with Chocolate, a previously proposed automatic design method that uses probabilistic finite state machines instead. In the first study, we assess Maple's ability to produce control software that crosses the reality gap satisfactorily. In the second study, we investigate Maple's performance as a function of the design budget, that is, the maximum number of simulation runs that the design process is allowed to perform. In the third study, we explore a number of possible variants of Maple that differ in the constraints imposed on the structure of the behavior trees generated. The results of the three studies indicate that, in the context of swarm robotics, behavior trees might be appealing but in many settings do not produce better solutions than finite state machines.

\section{INTRODUCTION}

In this paper, we extend the original definition of AutoMoDe-the family of automatic modular design methods proposed by Francesca et al. (2014) — to study the use of behavior trees as a control software architecture for robot swarms.

In swarm robotics, a large group of autonomous robots cooperate to perform a mission that is beyond the limited capabilities of a single robot (Beni, 2004; Şahin, 2004; Brambilla et al., 2013; Garattoni and Birattari, 2016). A robot swarm is highly redundant, self-organized, and decentralized in nature. These properties are appealing in applications that, for example, imply a high risk of individual failure, take place in locations with limited communication infrastructure, or require scalability (Dorigo et al., 2014). Unfortunately, these properties have also a downside: it is difficult to conceive and implement control software for the individual robots so that a desired collective behavior is produced. As a general methodology is still missing, the design process is typically labour intensive, time consuming, error prone, and difficult to reproduce (Brambilla et al., 2013; Francesca and Birattari, 2016; Bozhinoski and Birattari, 2018).

Automatic design is a valid and promising alternative (Francesca and Birattari, 2016; Birattari et al., 2019). In automatic design, the problem of designing control software to perform a given mission is re-formulated into an optimization problem: an optimization algorithm searches a space of candidate solutions so as to maximize an objective function. In this context, a candidate solution is an instance of the control software to be executed by each robot; and the objective function is a mission-dependent score that measures the performance of the swarm on the given mission. Because the evaluation of candidate 
solutions on physical robots is costly and time consuming, automatic design methods typically rely on simulation. ${ }^{1}$ A major issue with the adoption of simulation in automatic design is the so called reality gap (Brooks, 1992; Jakobi et al., 1995): the difference between simulation and reality, which is ultimately unavoidable. As a result of the reality gap, it is possible, and even likely, that control software generated in simulation suffers from a drop in performance when deployed in reality. The reality gap is one of the most challenging issues in the automatic design of robot swarms (Francesca and Birattari, 2016).

Evolutionary swarm robotics (Trianni, 2008, 2014) - the application of evolutionary robotics (Lipson, 2005; Floreano et al., 2008) to robot swarms-is a popular automatic design approach. In evolutionary swarm robotics, an evolutionary algorithm (Bäck et al., 1997) generates the control software of the robots, typically in the form of an artificial neural network. The input of the artificial neural network are the sensor readings; the output are the control actions that drive the actuators. Although evolutionary swarm robotics has been successfully used to generate control software for various missions (Quinn et al., 2003; Christensen and Dorigo, 2006; Hauert et al., 2009; Trianni and Nolfi, 2009), it presents some known limitations, among which is its inability to cross the reality gap reliably (Silva et al., 2016). Francesca et al. (2014) conjectured that the issues encountered by evolutionary robotics with the reality gap are due to the high representational power of artificial neural networks. This leads the design process to overfit characteristics of the simulator that do not have a counterpart in reality. As a result, the control software produced fails to generalize to the real world.

Inspired by the notion of bias-variance tradeoff (Geman et al., 1992) from the supervised learning literature, Francesca et al. (2014) developed AutoMoDe: an automatic design approach in which control software is conceived by automatically assembling predefined modules (that is, low-level behaviors and conditions) into a modular software architecture. The rationale behind AutoMoDe is to lower the representational power - and therefore the variance - of the control software it produces by introducing bias: it is restricted to be combinations of predefined modules. This restriction restrains the space of the possible instances of control software that can be generated by AutoMoDe, with the intent of reducing the risk to overfit characteristics of the simulator that are not a faithful representation of reality.

AutoMoDe is an abstract approach that, in order to be used, must be specialized to a specific robotic platform by defining low-level behaviors and conditions, the specific rules/constraints to combine them, and the optimization algorithm to search the space of solutions. So far, all instances of AutoMoDethat is, Vanilla (Francesca et al., 2014), Chocolate (Francesca et al., 2015), Gianduja (Hasselmann et al., 2018b), Waffle (Salman et al., 2019), Coconut (Spaey et al., 2019), Icepop (Kuckling et al., 2019), and TuttiFrutti (Garzón Ramos and Birattari, 2020) — that have been proposed target the e-puck robot (Mondada et al., 2009). To substantiate their conjecture, Francesca et al. (2014) compared the performance of Vanilla and Chocolate with EvoStick, an implementation of the classical evolutionary swarm robotics approach. In their experiments, Francesca et al. $(2014,2015)$ observed that both Vanilla and Chocolate are able to generate control software that crosses the reality gap satisfactorily. In addition, they observed what can be called a rank inversion (Ligot and Birattari, 2018, 2019): EvoStick outperforms Vanilla and Chocolate in simulation, but Vanilla and Chocolate outperform EvoStick in reality.

In the original definition, Francesca et al. (2014) have characterized AutoMoDe as an approach to generate control software in the form of a probabilistic finite-state machine (Francesca et al., 2014, 2015). However, this characterization appears to be too restrictive: the element that truly characterize AutoMoDe - whose name is the contraction of automatic modular design-is that it generates control software by combining and fine-tuning predefined modules. Indeed, according to the conjecture of Francesca et al. (2014), its modular nature is the main reason why AutoMoDe has shown to be robust to the reality gap: the architecture into which the modules are assembled appears to be a secondary issue.

In this paper, we aim at investigating the possibilities, challenges, and limitations that arise from the use of behavior trees in the context of the automatic modular design of collective behaviors in swarm robotics. Behavior trees are a popular control architecture originally proposed for game development (Champandard, 2007; Champandard et al., 2010), and offer a number of advantages over finite-state machines, such as enhanced expressiveness, inherent modularity, and two-way control transfers (Colledanchise and Ögren, 2018). Moreover, Colledanchise and Ögren (2018) have shown that behavior trees generalize a number of

${ }^{1}$ For the sake of completeness, we mention here that some automatic design methods do not rely on simulation. They operate while robots are deployed in their operating environment. We refer the reader to Francesca and Birattari (2016) for a discussion of advantages and limitations of these methods. 
other architectures including the subsumption architecture (Brooks, 1986) and decision trees (Nehaniv and Dautenhahn, 2002). Recently, behavior trees have attracted interest from the domains of artificial intelligence and robotics (Colledanchise and Ögren, 2018).

The main characteristics of behavior trees is the use of complex behavioral modules as leaf nodes that return their state of execution: running, success, or failure. Behavior trees are therefore a convenient way to implicitly model plans of execution: they define what action needs to be taken if a given condition is met or not, and if a given behavior succeeds or fails. The current practice of swarm robotics goes against the principle of planning as the individual robots used are typically simple and reactive in the sense defined by Brooks (1991). In the reactive paradigm, a low-level behavior is executed indefinitely until an external event triggers the indefinite execution of another low-level behaviors, and so on. Due to this cultural legacy, the low-level behaviors typically operated by robots within swarms do not have natural termination criteria, and therefore do not have success/failure states. In addition, the hardware limitations of the simple individual robots typically used in swarm robotics does not give them the capabilities of assessing natural termination criteria of the low-level behaviors they are executing. It is nonetheless possible to use behavior trees as a control software architecture for robot swarms, as it has already been done by Jones et al. (2018). However, to do so, design choices are needed and possibly only a subset of the functionalities of the behavior trees can be used, which forces one to renounce the implicit planning that they offer. For example, Jones et al. (2018) considered atomic commands as action nodes (i.e., move forward, turn left/right, or store data) that always return success after the second execution of the behavior, and never return failure. Despite not benefiting from the full potential of behaviors trees when combining low-level behaviors without natural termination criteria, it remains that the inherent modularity that they offer makes behavior trees a control architecture that is well worth exploring in the context of automatic design of robot swarm. Indeed, because each subtree is a valid structure, behavior trees are more easily manipulated than finite-state machines (Colledanchise and Ögren, 2018). Therefore, one could conceive tailored optimization algorithms based on local manipulations that explore the possible collective behaviors obtained by selecting, combining, and fine-tuning predefined modules into behavior trees more efficiently than into finite state machines.

In this work, we study the use of behavior trees in fully automatic off-line design of robot swarms (Birattari et al., 2020). We do so by developing a method that uses low-level behaviors that are more complex than those of Jones et al. (2018), but yet less complex than those typically used in applications of behaviors tree to other domains. Indeed, rather than using atomic commands and assuming the artificial return of success after a given time, we use low-level behaviors as they are typically conceived in swarm robotics, that is, without the notion of success or failure. We devised Maple, a novel instance of AutoMoDe that has at its disposal the same low-level behaviors and conditions used by Vanilla and Chocolate, with the goal of understanding the conditions under which it is beneficial to adopt behavior trees over finite state machines in modular automatic design. Maple is in many aspects similar to Chocolate: in fact, we only substituted probabilistic finite state machines with behavior trees. This way, differences in performance between the two methods can only be attributed to the different control architecture they adopt. Because the behavioral modules adopted in Maple only return running, Maple produces control software in the form of behavior trees with predetermined structure that only use a subset of the behavior trees functionalities. In this structure, a conditional module is combined with a low-level behavior in order to act as a termination criterion for the said low-level behavior. We present three empirical studies conducted on two missions. In the first one, we study the robustness of automatically generated control software in the form of behavior trees by comparing its performance in simulation and in reality. The results show that the control software generated by Maple performs similarly to the one generated by Chocolate, and that it crosses the reality gap more satisfactorily than the one generated by EvoStick. This confirms Francesca et al. (2014)'s conjecture that AutoMoDe is robust to the reality gap due to its modular nature. In the second study, we investigate the impact of different design budgets on the performance of the control software produced by Maple and Chocolate. The results indicate that Maple converges to satisfactory solutions faster than Chocolate. However, it appears that the expressiveness of the control structure adopted in Maple is reduced with regard to the one of finite state machines: Maple cannot produce solutions as complex as those produced by Chocolate for one of the missions considered. In the third study, we explore multiple alternatives to the structure of the behavior trees adopted in Maple. All these alternatives are predefined, restricted behavior trees structures that can be used with low-level behaviors that do not have a natural termination criterion. The results show that none of the explored 
structures outperform the one adopted in Maple in both missions considered. This paper extends on preliminary results presented in a conference (Kuckling et al., 2018a). We present here the complete description of the automatic design method and justifications of the design choices, together with more experimental results.

The work of Jones et al. (2018) brought initial evidence that behavior trees are a viable control architecture to be adopted in swarm robotics when considering atomic commands as action nodes. Our studies highlight the strengths and weaknesses of behavior trees when applied low-level behaviors as they are typically conceived in this domain: our results suggest that, although behavior trees might be appealing under some settings, under other they do not produce better results than finite state machines and might be even outperformed by the latter. What hinders the application of behavior trees to swarm robotics is the absence of the notion of success and failure in the low-level behaviors typically adopted in swarm robotics. We believe that, in order to develop low-level behaviors that are appropriate for behavior trees, one should overcome technical issues (that is, use robots whose hardware capabilities enable them to infer natural termination criteria) and a cultural legacy.

\section{BEHAVIOR TREES}

In this section, we give a brief description of behavior trees and their functioning. We adopt the framework that Marzinotto et al. (2014) proposed to unify the different variants of behavior trees described in the literature. We refer the reader to the original description of the framework for more details.

The original idea of behavior trees was proposed for the Halo 2 video game (Isla, 2005). Since then, behavior trees have found applications in many computer games, for example, Spore and Bioshock (Champandard et al., 2010). Recently, behavior trees have attracted the interest of the research community. Initial research focused on the automatic generation of behaviors in video games, for example, the commercial game DEFCON (Lim et al., 2010) and the Mario AI competition (Perez et al., 2011). Even more recently, behavior trees have found applications in the control of unmanned aerial vehicles (Ögren, 2012), surgical robots (Hu et al., 2015), and collaborative robots (Paxton et al., 2017).

A behavior tree is a control architecture that can be expressed as a directed acyclic graph with a single root. With a fixed frequency, the root generates a tick that controls the execution. The tick is propagated through the tree and activates each node that it visits. The path that the tick takes through the tree is determined by the inner nodes, which are called control-flow nodes. Once the tick reaches a leaf node, a condition is evaluated or an action is performed. Then, the leaf node immediately returns the tick to its parent together with one of the following three values: success, failure, or running. A condition node returns success, if its associated condition is fulfilled; failure, otherwise. An action node performs a single control step of its associated action and returns success, if the action is completed; failure, if the action failed; running, if the action is still in progress. When a control-flow node receives a return value from a child, it either immediately returns this value to its parent, or it continues propagating the tick to the remaining children. There are six types of control-flow nodes:

Sequence $(\rightarrow)$ : ticks its children sequentially, starting from the leftmost child, as long as they return success. Because it does not remember the last child that returned running, it is said to be memory-less. Once a child returns running or failure, the sequence node immediately passes the returned value, together with the tick, to its parent. If all children return success, the node also returns success.

Selector (?) : memory-less node that ticks its children sequentially, starting from the leftmost child, as long as they return failure. Once a child returns running or success, the selector node immediately passes the returned value, together with the tick, to its parent. If all children return failure, the node also returns failure.

Sequence* $\left(\rightarrow^{*}\right)$ : version of the sequence node with memory: resumes ticking from the last child that returned running, if any.

Selector* $^{*}\left(?^{*}\right)$ : version of the selector node with memory: resumes ticking from the last child that returned running, if any.

Parallel ( $\rightrightarrows)$ : ticks all its children simultaneously. It returns success if a defined fraction of its children return success; failure if the fraction of children return failure; running otherwise.

Decorator $(\delta)$ : is limited to a single child. It can alter the number of ticks passed to the child and the return value according to a custom function defined at design time.

In the context of automatic modular design, the most important properties of behavior trees are their enhanced expressiveness, the principle of two-way control transfers, and their inherent modularity (Ögren, 
Table 1. Reference model RM 1.1 (Hasselmann et al., 2018a). Sensors and actuators of the e-puck robot. Period of control cycle: $100 \mathrm{~ms}$.

\begin{tabular}{lrr}
\hline sensor/actuator & variables & values \\
\hline proximity & prox $_{i}$, with $i \in\{0, \ldots, 7\}$ & {$[0,1]$} \\
light & light $_{i}$, with $i \in\{0, \ldots, 7\}$ & {$[0,1]$} \\
ground & ground $_{i}$, with $i \in\{0, \ldots, 2\}$ & $\{$ black, gray, white $\}$ \\
range-and-bearing & $n$ & $\{0, \ldots, 19\}$ \\
& $V_{d}$ & $([0,0.7] \mathrm{m},[0,2 \pi]$ radian $)$ \\
wheels & $v_{l}, v_{r}$ & {$[-0.12,0.12] \mathrm{m} \mathrm{s}^{-1}$} \\
\hline
\end{tabular}

2012; Colledanchise and Ögren, 2018). Ögren and coworkers have shown that behavior trees generalize finite-state machines only with selector and sequence nodes (Ögren, 2012; Marzinotto et al., 2014). With parallel nodes, behavior trees are able to express individual behaviors that have no representation in classical finite-state machines. The principle of two-way control transfers implies that the control can be passed from a node to its child, and can also be returned from the child, along with information about the state of the system. Finally, behavior trees are inherently modular: each subtree is a valid behavior tree. Due to this property, behavior trees can be easily manipulated as one can move, modify, or prune subtrees without compromising the structural integrity of the behavior tree. The modularity of behavior trees could simplify the conception of tailored optimization algorithm based on local manipulations.

\section{AUTOMODE-MAPLE}

Maple is an automatic modular design method that generates control software in the form of behavior trees. It does so by selecting, combining, and fine-tuning a set of predefined modules: the six lowlevel behaviors and the six conditions defined by Francesca et al. (2014) for Vanilla, and later used in Chocolate (Francesca et al., 2015). We introduce Maple with the purpose of exploring the use of behavior trees as a control architecture in the automatic modular design of robot swarms. To conduct a meaningful study on the potentials of behavior trees as a control architecture, we compare Maple with Chocolate, a state-of-the-art automatic modular design method that generates control software in the form of probabilistic finite-state machines (Francesca et al., 2015; Francesca and Birattari, 2016). We conceived Maple to be as similar as possible to Chocolate so that differences in performance between the two methods can only be attributed to the different control architecture they adopt. Maple and Chocolate generate control software for the same robotic platform, they have at their disposal the same set of modules, and they use the same optimization algorithm.

In a probabilistic finite-state machine generated by Chocolate, a state is an instantiation of a low-level behavior and a transition is an instantiation of a condition. Because low-level behaviors (the states of the finite-state machine) are executed until an external condition (a transition) is enabled, they do not have inherent termination criteria. The absence of natural termination criteria implies that, when used as action nodes in a behavior tree generated by Maple, the low-level behaviors of Chocolate can only return running. As a result, part of the control-flow nodes of behavior trees do not work as intended. With Maple, we chose to use the unmodified modules of Chocolate, and force the generated behavior trees to adopt a restricted structure that only uses a subset of the control-flow nodes.

\section{Robotic platform}

Maple produces control software for the e-puck robot (Mondada et al., 2009) equipped with several extension boards (Garattoni et al., 2015), including the range-and-bearing board (Gutiérrez et al., 2009). The predefined modules on which Maple operates have access to a subset of the capabilities of the e-puck robot that are formally defined by the reference model RM 1.1 (Hasselmann et al., 2018a)—see Table 1.

The modules can adjust the velocity of the two wheels $\left(v_{l}\right.$ and $\left.v_{r}\right)$ of the robot, detect the presence of obstacles $\left(\operatorname{prox}_{i}\right)$, measure the intensity of the ambient light $\left(l i g h t_{i}\right)$, and identify whether the ground situated directly beneath the robot is black, gray, or white $\left(\right.$ ground $\left._{i}\right)$. The modules have also access to the number $n$ of surrounding peers within a range of up to $0.7 \mathrm{~m}$, as well as to a vector $V_{d}=\sum_{m=1}^{n}\left(1 / r_{m}, \angle b_{m}\right)$ where $r_{m}$ and $\angle b_{m}$ are distance and bearing of the $m$-th neighboring peer (Spears et al., 2004). 


\section{Set of modules}

Maple has at its disposal the same set of modules used by Vanilla (Francesca et al., 2014) and Chocolate (Francesca et al., 2015). Some of the modules are parametric so that the optimization algorithm can fine-tune their behavior on a per-mission basis. The set comprises six low-level behaviors and six conditions. A low-level behavior is a way in which the robot operates its actuators in response to the readings of its sensors. A condition is a context that the robot perceives via its sensors. Conditions contribute to determine which behavior is executed at any moment in time.

In the behavior trees generated by Maple, an action node is selected among the six low-level behaviors and a condition node is selected among the six conditions. In the following, we briefly describe the low-level behaviors and conditions. For the details, we refer the reader to their original description given by Francesca et al. (2014).

\section{Low-level behaviors}

Exploration: if the front of the robot is clear of obstacles, the robot moves straight. When an obstacle is perceived via the front proximity sensors, the robot turns in-place for a random number of control cycles drawn in $\{0, \ldots, \tau\} . \tau$ is an integer parameter $\in\{0, \ldots, 100\}$.

Stop: the robot does not move.

Phototaxis: the robot moves towards the light source. If no light source is perceived, the robot moves straight while avoiding obstacles.

Anti-phototaxis: the robot moves away from the light source. If no light source is perceived, the robot moves straight while avoiding obstacles.

Attraction: the robot moves towards its neighboring peers, following $\alpha V_{d}$, where the parameter $\alpha \in[1,5]$ controls the speed of convergence towards them. If no peer is perceived, the robot moves straight while avoiding obstacles.

Repulsion: the robot moves away from its neighboring peers, following $-\alpha V_{d}$, where the parameter $\alpha \in[1,5]$ controls the speed of divergence. If no peer is perceived, the robot moves straight while avoiding obstacles.

\section{Conditions}

Black-floor: true with probability $\beta$, if the ground situated below the robot is perceived as black.

Gray-floor: true with probability $\beta$, if the ground situated below the robot is perceived as gray.

White-floor: true with probability $\beta$, if the ground situated below the robot is perceived as white.

Neighbor-count: true with probability $z(n)=\left(1+e^{\eta(\xi-n)}\right)^{-1}$, where $n$ is number of detected peers. The parameters $\eta \in[0,20]$ and $\xi \in\{0, \ldots, 10\}$ control the steepness and the inflection point of the function, respectively.

Inverted-neighbor-count: true with probability $1-z(n)$.

Fixed-probability: true with probability $\beta$.

\section{Control software architecture}

The low-level behaviors of Chocolate have no inherent success or failure criterion and can only return running when used as action nodes in behavior trees. To use Chocolate's low-level behaviors as action nodes, we constrained Maple to generate behavior trees that have a particular, restricted structure. This restricted structure only uses a subset of the control-flow nodes of the classical implementation of behavior trees. The top-level node is a sequence* node $\left(\rightarrow^{*}\right)$ and can have up to four selector subtrees attached to it. A selector subtree is composed of a selector node (?) with two leaf nodes: a condition node as the left leaf node, and an action node as the right leaf node. Figure 1 illustrates a behavior tree with the restricted structure adopted here. We limit the maximal number of subtrees, and therefore the number of action nodes, to four so as to mimic the restrictions of Chocolate, which generates probabilistic finite-state machines with up to four states. 


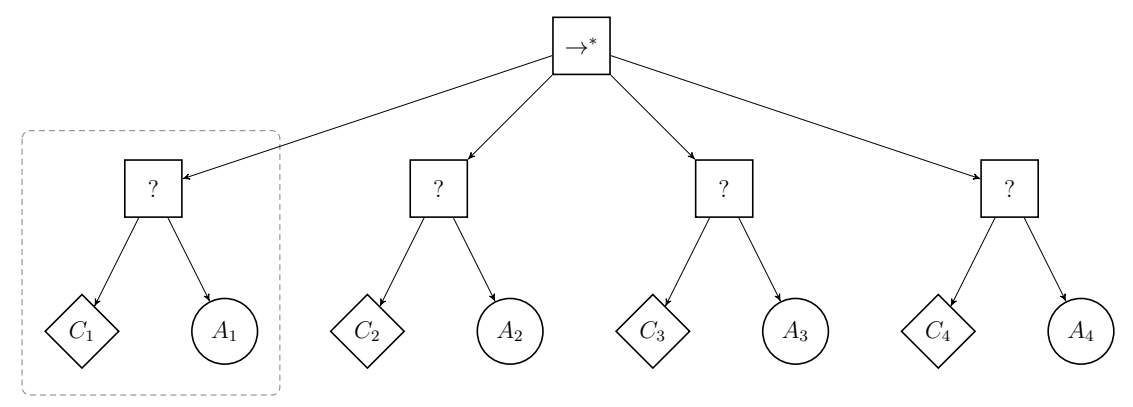

Figure 1. Illustration of a behavior tree with restricted structure that Maple can produce. Maple generates a behavior tree by defining first the number of selector subtrees (highlighted by the dashed box), and by then specifying and fine-tuning the condition and action nodes that compose each selector subtree.

In the example of Figure 1, the left-most selector subtree (highlighted by the dashed box) is first ticked and action $A_{1}$ is executed as long as condition $C_{1}$ returns failure. If condition $C_{1}$ returns success, the top-level node $\left(\rightarrow^{*}\right)$ ticks the second selector subtree, and $A_{2}$ is executed, provided that $C_{2}$ returns failure. Because the top-level node is a control-flow node with memory, the tick will resume at the second subtree in the following control cycle. $A_{2}$ is therefore executed as long as $C_{2}$ returns failure. Although actions $A_{1}$ and $A_{4}$ are not in adjacent sub-trees, $A_{4}$ can be executed directly after $A_{1}$ granted that conditions $C_{1}$, $C_{2}$, and $C_{3}$ return success and $C_{4}$ returns failure. When condition $C_{4}$ of the last selector subtree returns success, the top-level node of the tree also returns success and no action is performed. In this case, the tree is ticked again at the next control cycle, and the top-level node ticks the left-most selector subtree again.

The size of the space spanning all possible instance of control software that can be produced by Maple is in $O\left(|\mathscr{B}|^{4}|\mathscr{C}|^{4}\right)$, where $\mathscr{B}$ and $\mathscr{C}$ are the sets of low-level behaviors and conditions, respectively (Kuckling et al., 2018b). The search space can be formally defined as:

$$
\left[T, \# N^{(2)}, N_{i}^{(2)}, \# L_{i}, L_{i j}, L_{i j}^{p}\right], \quad \text { with } i=\left\{1, \ldots, \# N^{(2)}\right\}, j=\left\{1, \ldots, \# L_{i}\right\},
$$

where $T \in\{$ sequence* $\}$ is the type of the top-level node; $\# N^{(2)} \in\{1, \ldots, 4\}$ is the number of level 2 nodes; $N_{i}^{(2)} \in\{$ selector $\}$ is the type of the level 2 node $i$; $\# L_{i} \in\{2\}$ is the number of leafs of node $i ; L_{i j}$ is the type of the $j$-th leaf of node $i$, with $L_{i 1} \in \mathscr{C}$ and $L_{i 2} \in \mathscr{B}$; and $L_{i j}^{p}$ are the parameters of leaf $L_{i j}$.

\section{Optimization algorithm}

Maple uses Iterated F-race (Balaprakash et al., 2007; López-Ibáñez et al., 2016) as an optimization algorithm. Iterated F-race searches the space of all possible candidate solutions for the best one according to a mission-specific measure of performance. The Iterated F-race algorithm comprises multiple steps, each of which is reminiscent of a race. In the first race, a uniformly distributed set of candidate solutions is sampled. These candidates are initially evaluated on a set of different instances. Typically, an instance describes the configuration of the arena at the beginning of an experiment (that is, positions and orientations of the robots, positions of eventual obstacles or objects of interest, or color of the floor). After the initial set of evaluations is performed, a Friedman test (Friedman, 1937, 1939; Conover, 1999) is performed on the performance obtained by the candidate solutions. The candidate solutions that perform significantly worse than at least another one are discarded. The algorithm keeps evaluating the remaining candidate solutions on new instances and discards those that are statistically dominated. The race terminates when only one surviving candidate solution remains, or when the maximal number of evaluation defined for the race is reached. In the following races, the new set of candidate solutions is sampled with a distribution that gives higher priority to solutions that are similar to the surviving solutions of the previous one.

\section{EXPERIMENTAL SETUP}

In this section, we describe the experimental setup that is common to the three studies conducted. In particular, we describe the previously proposed automatic design methods against which we compare Maple, the missions for which we generate control software, and the protocol we follow. Further details are given in each of the sections dedicated to the specific studies. 

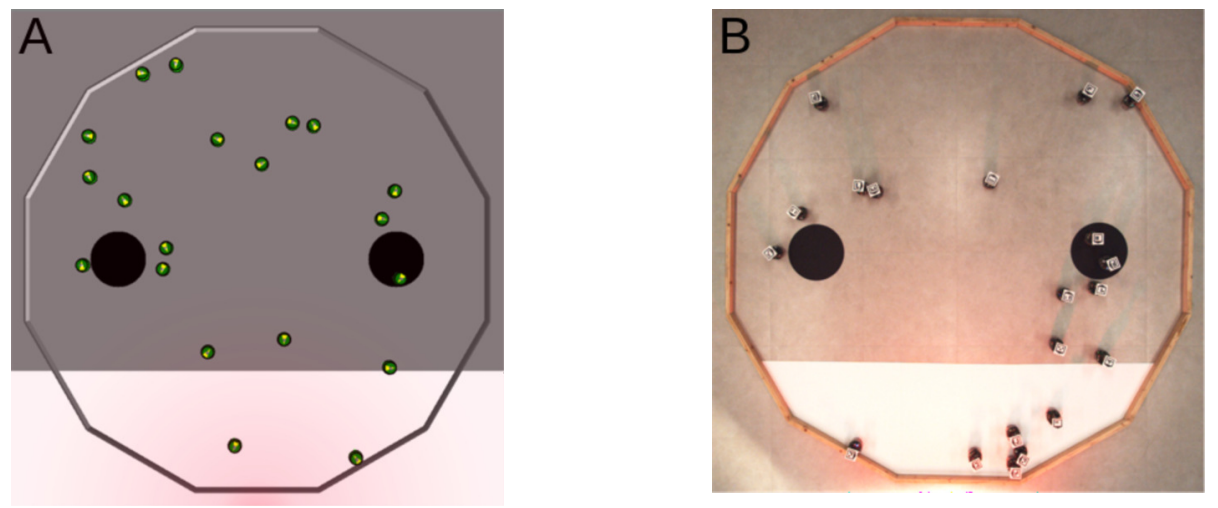

Figure 2. Foraging. (A) Simulated arena. (B) Real arena. The red glow visible in the picture is due to a red gel we placed in front of the light source. With the red gel, the light does not disturb the overhead camera that is used to track the position of the robots and compute the objective function. Yet, the light is still perceived by the robots that use their infrared sensors to sense it.

\section{Automatic design methods}

In Study 1 and 2, we compare Maple with two previously proposed methods: Chocolate and EvoStick. Maple is described in the previous section. Here, we briefly describe Chocolate and EvoStick: we refer the reader to Francesca et al. $(2014,2015)$ for the details.

Chocolate (Francesca et al., 2015) is an automatic modular method that selects, combines, and fine-tunes the same twelve predefined modules as Maple. In Chocolate, the architecture of the control software is a probabilistic finite-state machine. In this context, a state is an instance of low-level behavior and an edge is an instance of condition. Similarly to Maple, Chocolate adopts Iterated F-race as an optimization algorithm. With Chocolate, the search space of Iterated F-race is restricted to probabilistic finite-state machines that comprise up to four states, and up to four outgoing edges per state. The size of the search space defined by the control architecture of Chocolate is in $O\left(|\mathscr{B}|^{4}|\mathscr{C}|^{16}\right)$, where $\mathscr{B}$ and $\mathscr{C}$ are the sets of low-level behaviors and of conditions, respectively (Kuckling et al., 2018b).

EvoStick (Francesca et al., 2014, 2015) is a straightforward implementation of the evolutionary swarm robotics approach. In EvoStick, the architecture of the control software is a fully-connected, single layer, feedforward neural network. The neural network comprises 24 input nodes for the readings of the sensors described in the reference model RM 1.1: 8 for the proximity sensors, 8 for the light sensors, 3 for ground sensors, and 5 for the range-and-bearing board. Out of the five input nodes dedicated to the range-and-bearing board, one is allocated to the number of detected peers and the four others are allocated to the scalar projection of the vector $V_{d}$ on four unit vectors. The neural network comprises 2 output nodes controlling the velocities of the wheels. The topology of the neural network is fixed, and an evolutionary algorithm fine-tunes the 50 weights of the connections between the input and the output nodes. Each weight is a real value in the range $[-5,5]$. In EvoStick, the population is composed of 100 individuals that are evaluated 10 times per generation.

\section{Missions}

We consider two missions: FORAGING and AGGREGATION. The two missions must be performed in a dodecagonal arena delimited by walls and covering an area of $4.91 \mathrm{~m}^{2}$. The swarm is composed of 20 e-puck robots that are distributed uniformly in the arena at the beginning of each experimental run, and we limit the duration of the missions to $120 \mathrm{~s}$.

FORAGING. Because the robots cannot physically carry objects, we consider an idealized form of foraging. In this version, we reckon that an item is picked up when a robot enters a source of food, and that a robot drops a carried item when it enters the nest. A robot can only carry one item at a time. In the arena, a source of food is represented by a black circle, and the nest is represented by the white area (see Figure 2). The two black circles have a radius of $0.15 \mathrm{~m}$, they are separated by a distance of $1.2 \mathrm{~m}$, and are located at $0.45 \mathrm{~m}$ from the white area. A light source is placed behind the white area to indicate the position of the nest to the robots. 

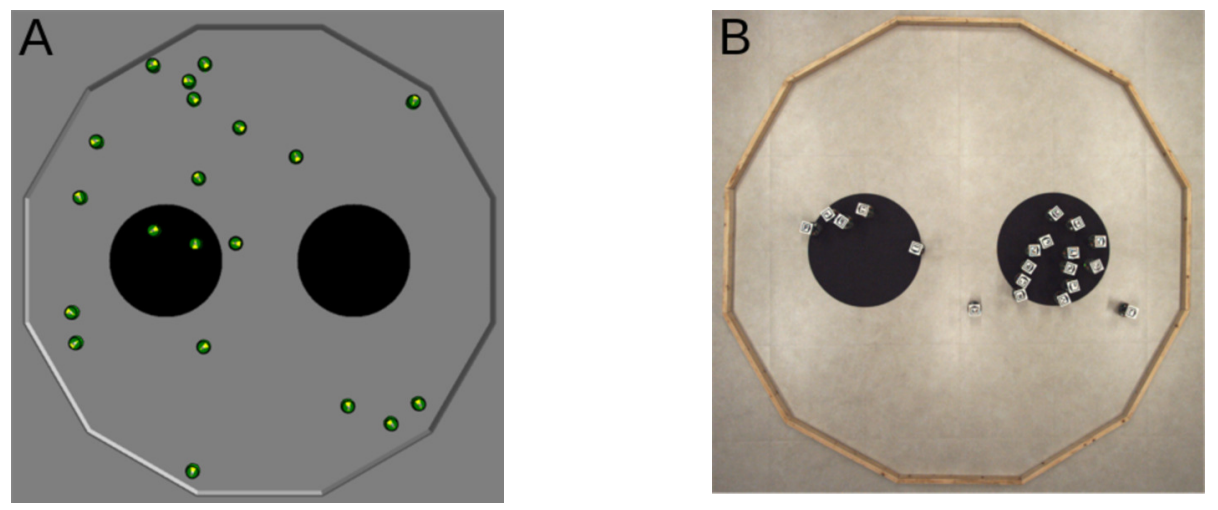

Figure 3. AGGREGATION. The objective function $F_{\mathrm{A}}$ is computed as the maximal fraction of robots situated either on the left area $\left(N_{l} / N\right)$ or on the right area $\left(N_{r} / N\right)$. It is evaluated at the end of an experimental run. (A) Simulated arena, with $F_{\mathrm{A}}=0.1$ as 2 robots stand on the left black area $\left(N_{l}=2\right)$ and no robot stands on the right one $\left(N_{r}=0\right)$. (B) Real arena, with $F_{\mathrm{A}}=0.65$ as $N_{l}=5$ and $N_{r}=13$.

The goal of the swarm is to retrieve as many items as possible from the sources to the nest. In other words, the robots must go back and forth between the black circles and the white area as many times as possible. The objective function is $F_{\mathrm{F}}=I$ where $I$ is the number of items deposited in the nest.

AGGREgation. The swarm must select and aggregate in one of the two black areas (see Figure 3). The two black areas have a radius of $0.3 \mathrm{~m}$ and are separated by a distance of $0.4 \mathrm{~m}$. The objective function is $F_{\mathrm{A}}=\max \left(N_{l}, N_{r}\right) / N$, where $N_{l}$ and $N_{r}$ are the number of robots located on the left and right black area, respectively; and $N$ is the total number of robot in the swarm. The objective function is computed at the end of a run and is maximized when all the robots have aggregated in the same black area.

\section{Dummy control software}

Throughout the three studies, we compare the performance of automatically generated control software to the one of two instances of control software - one per mission — that we call "dummy" control software. They perform a simple, naive, and trivial behavior that we can consider as a baseline for each mission. With this comparison, we assess whether the automatic design methods can produce behaviors that are more sophisticated than trivial solutions. To produce the two instances of dummy control software, we used the same low-level behaviors and conditions that Maple and Chocolate have at their disposal to generate control software. For FORAGING, we consider a strategy in which the robots move randomly in the environment. We obtained this strategy by using the low-level behavior exploration. For AGGREGATION, we consider a strategy in which the robots explore the environment randomly, and stop when they encounter a black spot. We obtained this strategy by combining the modules exploration, black-floor, and stop. To fine-tune the parameters of the modules, we used Iterated F-race with a design budget of $1 \mathrm{k}$ simulation runs.

\section{Methodology}

To account for the stochasticity of the design process, we execute each design method several times, and therefore produce several instances of control software. The number of executions of the design methods varies with the study. To evaluate the performance of a design method, each instance of control software is executed once in simulation. In Study 1, each instance of control software is also executed once in reality.

Simulations are performed with ARGoS3, version beta 48 (Pinciroli et al., 2012; Garattoni et al., 2015). In the experiments with the robots, we use a tracking system comprising an overhead camera and QR-code tags on the robots to identify and track them in real time (Stranieri et al., 2013). With this tracking system, we automatically measure the performance of the swarm, and we automatically guide the robots to the initial position and orientation for each evaluation run. During an evaluation run, the robots may tip over due to collisions. To avoid damages, we intervene to put them upright.

In the three studies, we present the performance of the design methods in the form of box-and-whiskers boxplots. In addition, we present the median performance of the dummy control software assessed in simulation with a dotted horizontal line. In each study, statements such as "method $A$ is significantly 

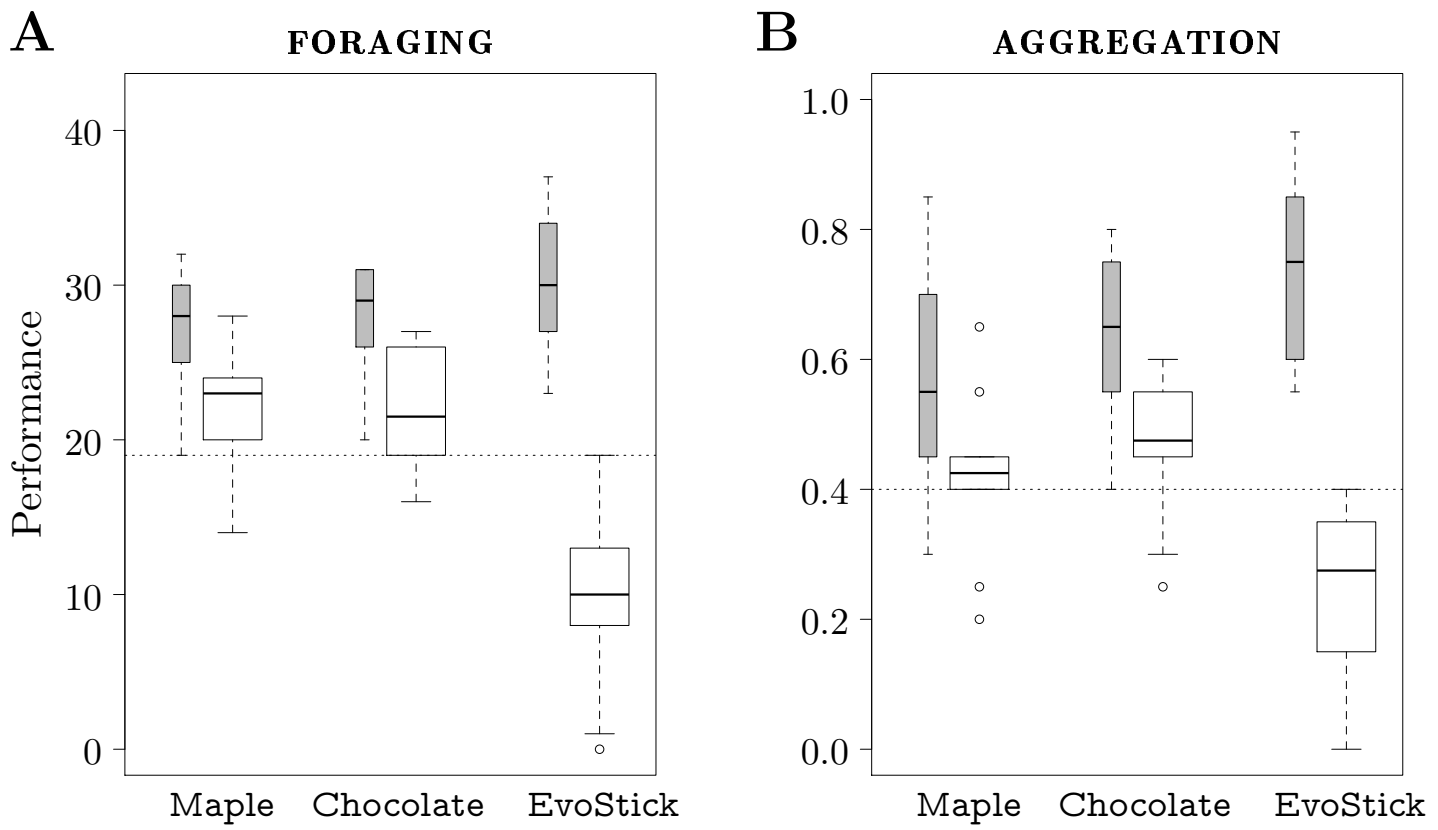

Figure 4. Results of Study 1. The gray boxes represent the performance assessed in simulation; the white boxes the one assessed in reality. The dotted line represents the median performance of the dummy control software assessed in simulation.

better/worse than $B$ " imply that significance has been assessed via a Wilcoxon rank-sum test, with confidence of at least $95 \%$. The instances of control software produced, the experimental data collected in simulation and in reality, and videos of the behavior displayed by the swarm of physical robots are available online as supplementary material (Ligot et al., 2020).

\section{STUDY 1: PERFORMANCE IN SIMULATION AND REALITY}

In this section, we evaluate Maple's ability to produce control software that crosses the reality gap satisfactorily. To do so, we compare the performance of control software generated by three design methodsMaple, Chocolate and EvoStick-both in simulation and in reality. Previous research (Francesca et al., 2015) indicates that Chocolate crosses the reality gap more satisfactorily than EvoStick. Francesca et al. $(2014,2015)$ argue that Chocolate's ability to cross the reality gap is mainly due to its modular nature. Because Maple shares with Chocolate the same modular nature and differs from it only in the control architecture adopted, we expect Maple to also experience smaller performance drops than EvoStick.

We executed each design method 10 times, and thus produced 10 instances of control software. The design budget allocated to each method is 50k simulation runs. The results are depicted in Figure 4.

FORAGING. In simulation, the performance of the control software produced by the three automatic design methods is similar, and is significantly better than the one of the dummy strategy. In reality, EvoStick is significantly worse than Maple and Chocolate. The performance of all three methods drops significantly when passing from simulation to reality, but EvoStick suffers from the effects of the reality gap the most. See Figure 4A.

Most of the instances of control software generated by Maple and Chocolate display similar strategies: the robots explore the environment randomly and once a black area (that is, a source of food) is found, they navigate towards the light to go back to the white area (that is, the nest). One instance of control software produced by Maple uses the anti-phototaxis low-level behavior to leave the nest faster once an item has been dropped. Three instances of control software produced by Chocolate display an even more sophisticated strategy: the robots only explore the gray area in the search for the sources of food. In other words, the robots always directly leave the nest if they enter it, independently of whether they dropped an item or not.

In simulation, the instances of control software generated by EvoStick display drastically different 
behaviors than the ones produced by Maple and Chocolate: the robots navigate following circular trajectories that cross at least one food source and the nest. In reality, the robots follow circular trajectories that are much smaller than those displayed in simulation. As a result, the robots tend to cluster near the light. Contrarily to Maple and Chocolate, and with the exception of few cases, the instances of control software generated by EvoStick do not display an effective foraging behavior.

AGGREgATION. In simulation, EvoStick performs significantly better than Maple and Chocolate, which show similar performance. In reality, we observe an inversion of the ranks: Maple and Chocolate perform significantly better than EvoStick. Indeed, the performance of EvoStick drops considerably, whereas the performance drop experienced by Maple and Chocolate is smaller. See Figure 4B.

The instances of control software produced by Maple and Chocolate efficiently search the arena and make the robots stop on the black areas once they are found. In simulation, with the control software produced by EvoStick, the robots follow the border of the arena and then adjust their trajectory to converge towards neighboring peers that are already situated on a black spot. In reality, the control software generated by EvoStick does not display the same behavior: robots are unable to find the black areas as efficiently as in simulation because they tend to stay close to the borders of the arena. Moreover, the robots tend to leave the black areas quickly when they are found. Although the three design methods perform significantly better than the dummy control software in simulation, none of the methods produced control software that makes the physical robots reach a consensus on the black area on which they should aggregate.

\section{STUDY 2: PERFORMANCE VERSUS DESIGN BUDGET}

In this section, we investigate the performance of Maple and Chocolate across different design budgets. Because the search space (that is, all instances of control software that can be generated) of Chocolate is significantly larger than the one of Maple- $O\left(|\mathscr{B}|^{4}|\mathscr{C}|^{16}\right)$ and $O\left(|\mathscr{B}|^{4}|\mathscr{C}|^{4}\right.$ ), respectively (Kuckling et al., 2018b) - we expect Maple to converge to high performing solutions faster than Chocolate.

We consider 6 design budgets: .5k, 1k, 5k, 10k, 50k and 200k simulation runs. For each design budget, we executed each design method 20 times, and thus produced 20 instances of control software. In total, the two design methods have been executed 120 times each. The results are depicted in Figure 5.

FORAGING. The performance of the methods show different trends when the design budget increases. For Maple, there is a significant improvement of the performance between design budgets of $1 \mathrm{k}$ and $5 \mathrm{k}$, and between 50k and 200k simulation runs. For Chocolate, the performance increases significantly between design budgets of $5 \mathrm{k}$ and $10 \mathrm{k}, 10 \mathrm{k}$ and $50 \mathrm{k}$, and 50k and 200k simulation runs. See Figure 5A.

With very small design budgets- .5k and $1 \mathrm{k}$ simulation runs-Maple and Chocolate show similar performance: they are unable to find solutions that are better than the dummy control software. With a small design budget $-5 \mathrm{k}$ simulation runs-Maple performs significantly better than Chocolate. Also, with $5 \mathrm{k}$ simulation runs, Chocolate and the dummy control software show similar performance. With a large design budget—200k runs-Chocolate performs significantly better than Maple. Indeed, the instances of control software generated by Chocolate display a more sophisticated foraging strategy than those generated by Maple: to increase the rate of discovery of the food sources, the robots only explore the gray area of the arena, and stay away from the nest. It appears that, with Maple's restrictions on the structure of the behavior trees, this strategy cannot be produced. Indeed, in the instances of control software that can be produced by Maple, only one condition can terminate the execution of the action performed, whereas in the ones produces by Chocolate, up to four conditions can. Therefore, with Maple, the robots are forced to explore the whole arena until they find the food sources (that is, the black circles). However, it is important to notice that the behavior trees generated by Maple with a design budget of $5 \mathrm{k}$ simulation runs are only outperformed by probabilistic finite-state machines when $200 \mathrm{k}$ simulation runs are allocated to Chocolate.

AGGREGATION. The performance of the control software generated by both methods increase almost constantly with the design budget. Also for this mission, Chocolate requires a design budget of at least $10 \mathrm{k}$ simulation runs in order to generate control software that is significantly better than the dummy control software. Contrarily, Maple only requires $1 \mathrm{k}$ simulation runs. With $1 \mathrm{k}$ and $5 \mathrm{k}$ simulation runs, Maple outperforms Chocolate. For larger design budgets, Maple and Chocolate show similar performance. See Figure 5B. 

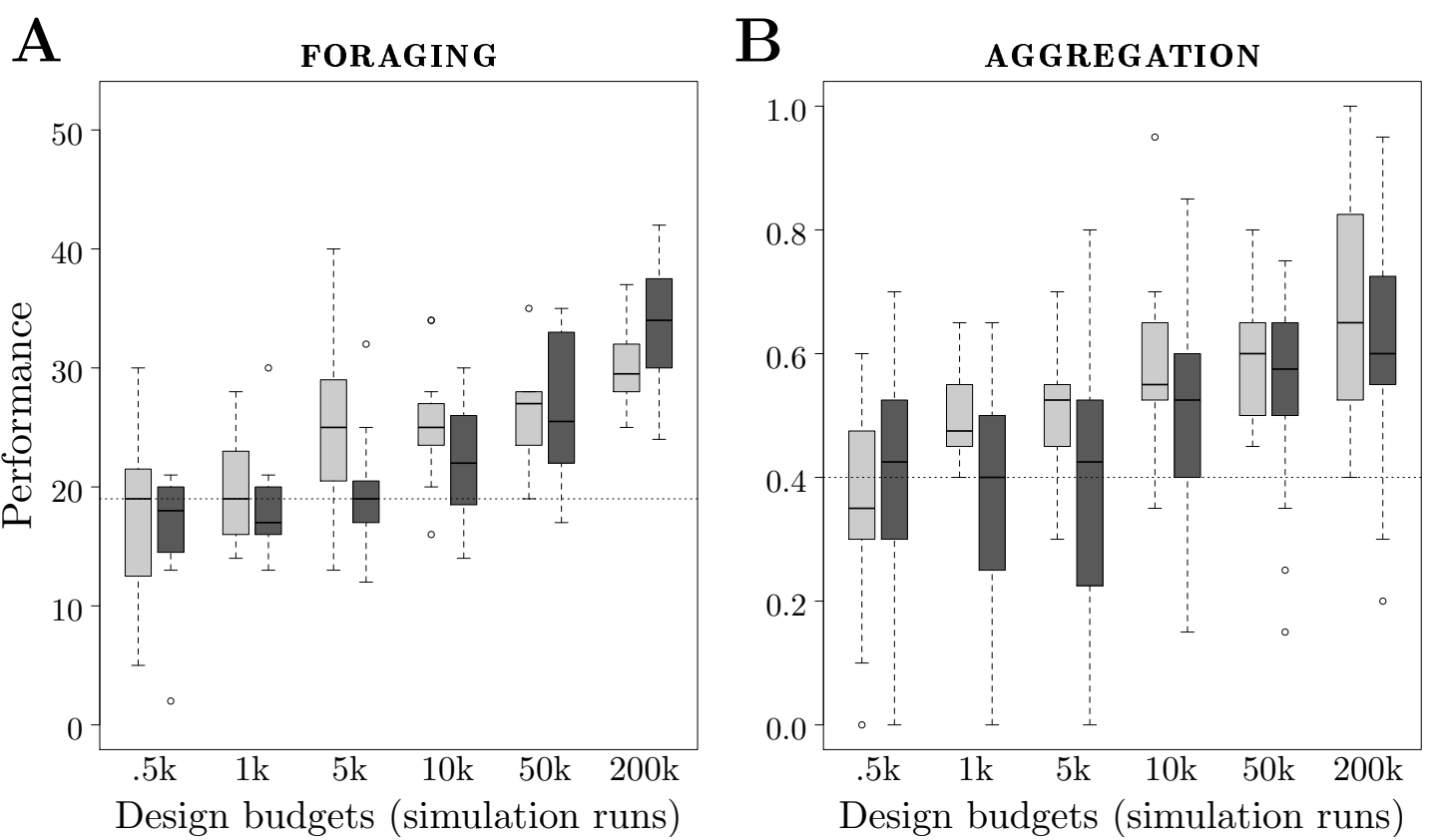

Figure 5. Results of Study 2. Performance of Maple and Chocolate over multiple design budgets, expressed in number of simulation runs. Light gray boxes represent the performance of Maple, dark gray boxes the one of Chocolate. The dotted line represents the median performance of the dummy control software.

Although the design budgets considered allow the two methods to outperform the dummy control software in multiple occasions, neither of them generated control software that completed the mission satisfactorily. Indeed, the maximal median performance obtained is $F_{\mathrm{A}}=0.65$, which means that only 13 out of the 20 robots were on the same black spot.

\section{STUDY 3: MAPLE AND SOME OF ITS POSSIBLE VARIANTS}

In this section, we explore the changes in performance when variations to the control architecture of Maple are introduced. Our exploration is not exhaustive: we only consider variants that generate behavior trees whose structure is similar to the one of the behavior trees generated by Maple. We limit our exploration to variants that generate trees with: (i) 3 levels (top-level, inner, and leaf nodes); (ii) up to 4 branches connected to the top-level node; and (iii) exactly 2 leaf nodes per branch. Many variants are possible, however, because the action nodes of Maple can only return running, some variants are unable to combine low-level behaviors into meaningful and elaborate individual behaviors. Descriptions of these variants, as well as illustrations, are given as part of supplementary material (Ligot et al., 2020). In the following, we describe variants that are promising and explain how they behave with the modules considered in Maple. We tested the most promising variants by generating control software and evaluating their performance in simulation, and report the results.

\section{Alternative behavior tree structures}

ICFN (inverted control-flow nodes): The control-flow nodes are inverted with regard to the ones of Maple: the top-level node is a selector* and the inner nodes are sequence nodes. See Figure 6A. In this variant, the action node of a subtree is executed as long as the condition returns success, whereas it is executed until the condition returns success in Maple.

ND (negation decorator): A negation decorator node can be instantiated above a condition node. See Figure 6B. The negation decorator returns failure (success) if the condition returns success (failure). With the set of conditions available, it is particularly interesting to place a negation decorator above a condition on the color of the ground perceived (that is black-, gray-, or white-floor). Indeed, placing a negation decorator node above a neighbor-count condition is equivalent to having an 
inverted-neighbor-count condition, and vice versa. Similarly, a negation decorator above a fixedprobability condition with $\rho$ is equivalent to a fixed-probability with $1-\rho$. However, a negation decorator above a condition on a given color is equivalent to assessing the conditions for the two other colors simultaneously.

FL (free leaves): Each leaf node is to be chosen between condition and action nodes. See Figure 6C. Four pairs of leaf nodes are possible: condition-condition (see first branch), condition-action (which corresponds to the leaf pair imposed in Maple, see second branch), action-condition (see third branch), and action-action (see fourth branch). For each subtree, the optimization algorithm is free to chose any pair of leaf nodes. The variant can express disjunction of conditions: a branch following a condition-condition leaf pair is ticked if the first or the second condition is met. However, the variant introduces dead-end states: when an action on the left hand side of a leaf pair is ticked, the action is executed for the remaining of the simulation run.

CA $\mid C C$ (condition-action or condition-condition): The right-hand side leaf node can be a condition or an action node. Two pairs of leaf nodes are thus possible: condition-action, condition-condition. With respect to FL, this variant can also express disjunction of conditions, but does not allow for dead-end states.

SP (success probability): Each action node has a probability $\rho$ to return success. The probability $\rho$ is a real value in the range $[0,1]$ and is tuned by the optimization process. With this probability, we simulate the capability of the action nodes to assess if the low-level behaviors are successfully executed.

\section{Results}

For each variant, we produced 20 instances of control software, all generated by the same optimization process-Iterated F-race-with a design budget of 50k simulation runs. We compare the performance of the variants to the one of Maple.

FORAGING. None of the variants outperformed Maple. Maple, ND, and CA|CC perform similarly; moreover, they outperform ICFN, FL, SP, and the dummy control software. The variants ICFN, FL, and the dummy control software show similar performance. See Figure 7A.

All the instances of control software generated by Maple show similar behaviors: the robots explore the arena until they find one of the food sources, then navigate towards the nest using the light as a guidance. In some cases, the robots use the anti-phototaxis low-level behavior to directly leave the nest once they have deposited an item.

With variant ND, we can manually design control software that displays an elaborate strategy: the robots increase the rate at which they discover food sources by only exploring the gray area of the arena. This behavior cannot be expressed by Maple (see Study 2). An example of a behavior tree adopting variant ND that displays this strategy is illustrated in Section 5 of the supplementary material (Ligot et al., 2020). In this example, the elaborate strategy only emerges if the success probability of the condition node below the negation decorator is set to 1 . Indeed, if the success probability is slightly lower, the behavior displayed is radically different, and more importantly, inefficient. It appears that, with the allocated budget, this necessary condition makes it unlikely for Iterated F-race to produce this strategy.

Iterated F-race was not able to take advantage of the disjunction of conditions that is available in $\mathrm{CA} \mid \mathrm{CC}$ to find better solutions that those of Maple. Indeed, we are unable to do so ourself. However, the increased search space of $\mathrm{CA} \mid \mathrm{CC}$ does not hinder the optimization process as results obtained are similar to those of Maple.

In variant SP, the success probabilities, together with the conditions, are termination mechanisms for the subtrees. The additional termination mechanisms makes it harder for Iterated F-race to exploit correlations between conditions and actions that lead to behaviors as efficient as those generated by Maple. Most of the produced control software rely essentially on the exploration low-level behavior.

With variant ICFN, one can generate a behavior tree that expresses the same elaborate strategies that can be generated with variant ND (see Section 5 of the supplementary material (Ligot et al., 2020) for an example). However, ICFN is faced with a similar problem as ND: the success probability of the conditions needs to be set to 1 in order for that elaborate strategy to emerge. With a success probability 


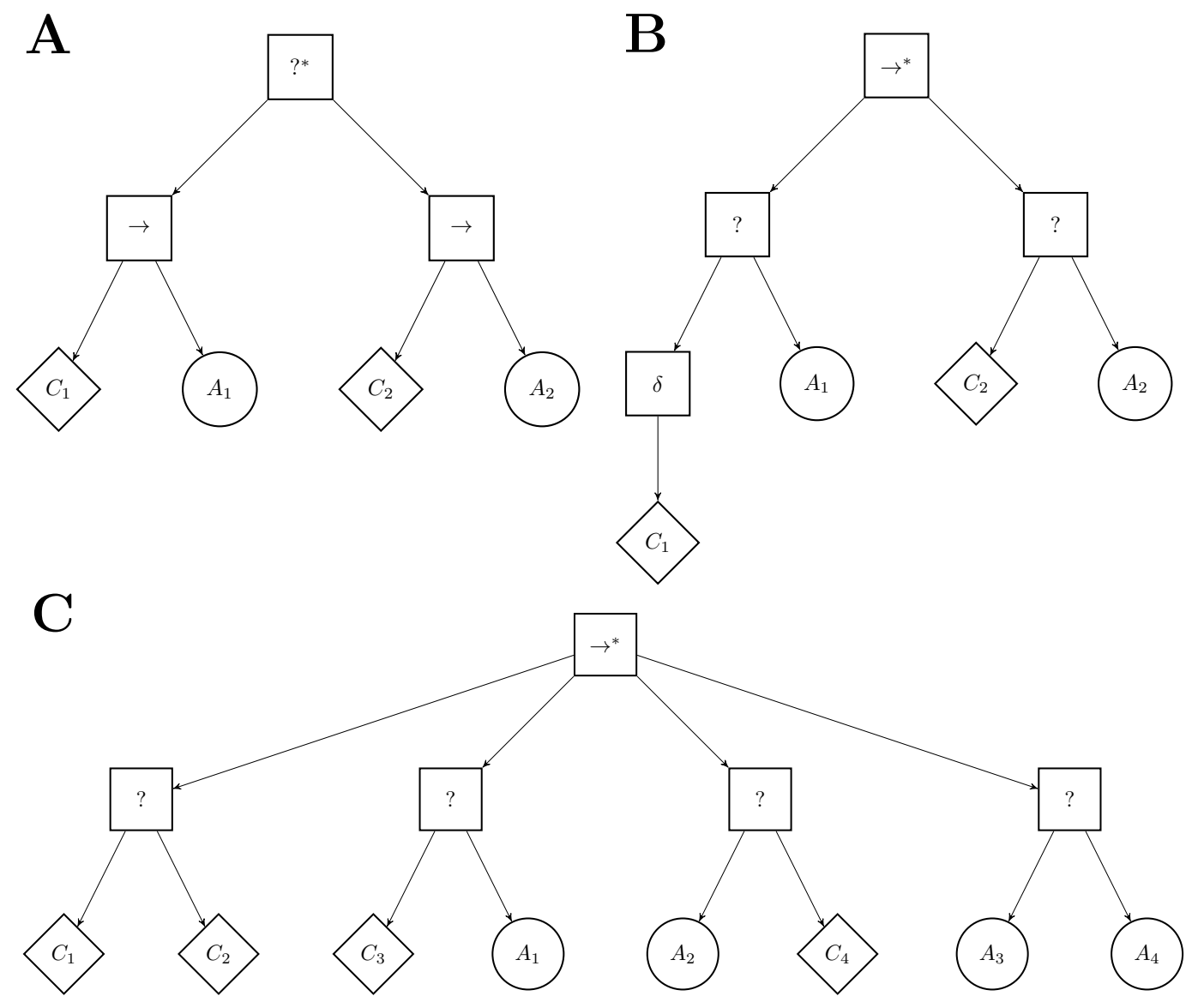

Figure 6. A few examples of Maple's variants. (A) variant ICFN (inverted control flow nodes), (B) variant ND (negation decorator), (C) variant FL (free leaves). The number of branches connected to the top-level node, and their order, has been chosen arbitrarily. 

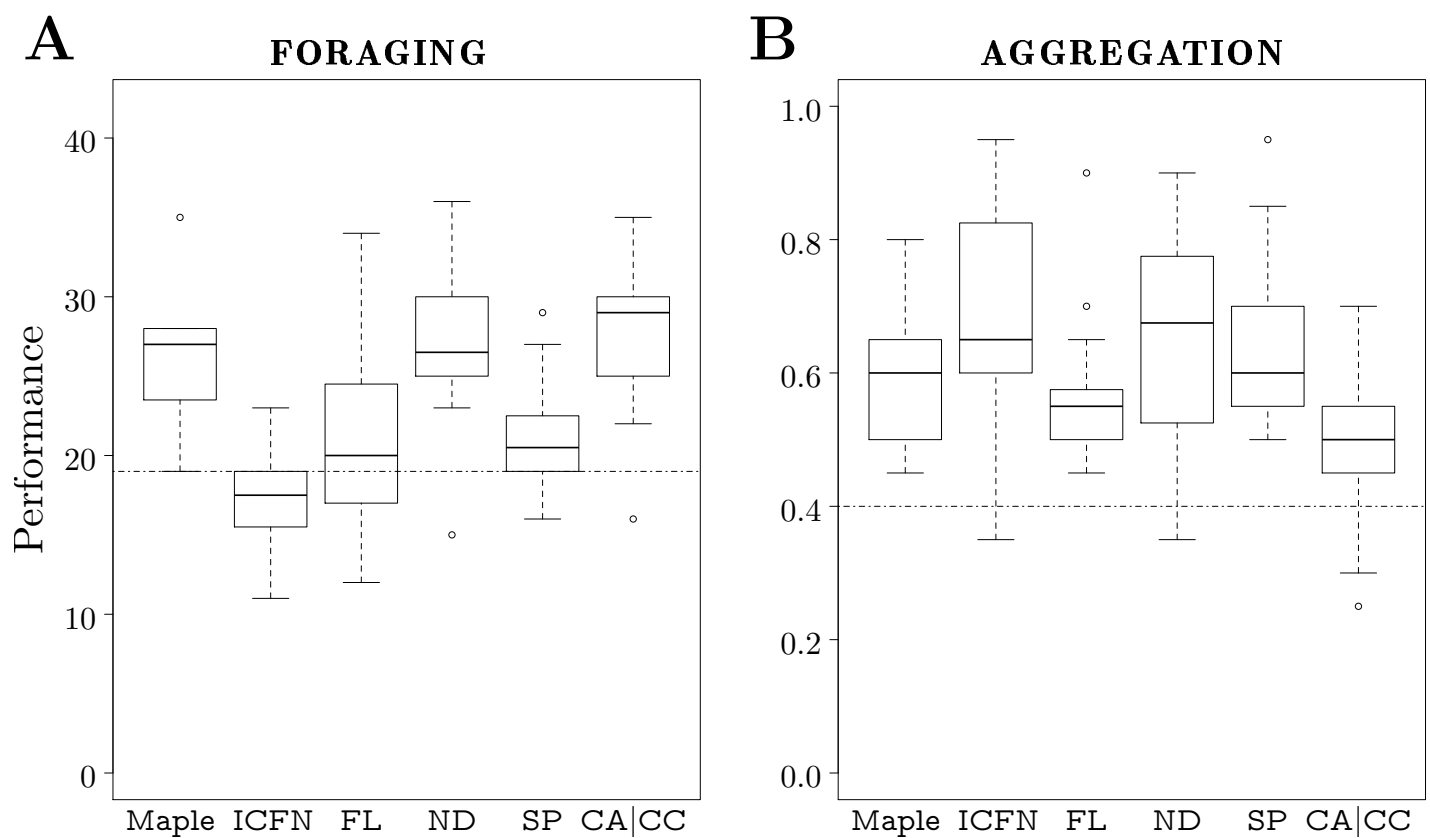

Figure 7. Results of Study 3. Performance in simulation of different variants of Maple. The dotted line represents the median performance of the dummy control software.

set to a lower value, the condition node might return failure even though its condition is met, and the subtree might therefore terminate prematurely. The allocated design budget was not large enough for Iterated F-race to find behavior trees with meaningful connections between the conditions and behaviors, which resulted in poor performance.

The performance of the variant FL shows the highest variance. Sometimes, the behavior trees generated are similar to those produced by Maple. However, in many cases, the left leaf node of subtrees is an action node with an associated exploration low-level behavior. Once this node is reached, this low-level behavior is executed until the end of the experimental run. As a result, the performance observed is similar to the one of the dummy control software.

AGGREGATION. Variant ICFN outperforms Maple. Maple, FL, ND, and SP show similar performance. Maple outperforms CA|CC. Every variant produced behavior trees that outperform the dummy control software. See Figure 7B.

All the instances of control software generated by Maple and the different variants make use of exploration and attraction as low-level behaviors to efficiently search for black spots. Maple and FL use stop as a low-level behavior in order to keep the robots on the discovered spot. Contrarily, the majority of the behavior trees adopting variant ICFN, ND, SP, and CA|CC do not contain the stop low-level behaviors as action nodes. Instead, they take advantage of the fact that, when no action node is executed, the robot stands still. ICFN is the only variant for which Iterated F-race was able to exploit this feature to outperform Maple.

\section{RELATED WORK}

Originating from game development (Isla, 2005), behavior trees have found recent applications in artificial intelligence (Perez et al., 2011) and robotics. Most of the robotics research focuses on their use in manipulators. Bagnell et al. (2012) used behavior trees to control a manipulator to perform simple manipulation tasks. Hu et al. (2015) used them to control the Raven-II surgical robot to perform an abstract version of tumor ablation surgery. Behavior trees have also been used as a control software for mobile robotics systems. Marzinotto et al. (2014) designed a behavior tree to make a NAO robot move towards a table and grasp an object. In all of the presented studies, behavior trees have been manually designed. 
To the best of our knowledge, the work of Jones et al. (2018) is the first and only application of behavior trees in the context of automatic off-line design of robot swarms ${ }^{23}$. The authors proposed a method based on genetic programming that automatically generates control software for a swarm of Kilobots in the form of behavior trees. The method has been tested on a foraging task in simulation and in reality. Their results suggest that behavior trees can be used as a control architecture in swarm robotics. Besides the different optimization processes adopted in the method of Jones et al. and in Maple, another major difference between the two methods lies in the action nodes used. In the method proposed by Jones et al., low-level behaviors are atomic commands: for example, move forward, turn left/right, or store data. Contrarily, the low-level behaviors that can be combined by Maple are more complex actions. Regardless of this difference, the low-level behaviors of the two methods lack natural success or failure termination criteria. To use their atomic low-level behaviors as action nodes in behavior trees, Jones et al. programmed the action nodes such that they return success after the second execution of the behavior, but failure is never returned. This solution allowed their method to have no restrictions on the selection of the control-flow nodes. In Maple, the action nodes can only return running, but the structure of the behavior trees, and the control-flow nodes, are restricted such that an external condition terminates the execution of an action.

\section{CONCLUSIONS}

In this paper, we presented Maple: an automatic modular design method that generates control software for robot swarms in the form of behavior trees. Maple is part of the AutoMoDe family: it generates control software by selecting, combining, and fine-tuning a set of predefined modules. Previous instances of AutoMoDe have all used probabilistic finite-state machines as a control architecture. In comparison to finite-state machines, behavior trees offer a number of appealing features. However, most of these features only emerge if the action nodes return their states of execution, that is, if the robot is able to tell whether the low-level behavior it executes is terminated successfully, could not execute normally, or still requires time to terminate. In the context of swarm robotics, the simple and reactive robots typically used are not able to determine the state of execution of the low-level behaviors they operate. With Maple, we have investigated the use of behavior trees as a control architecture in the automatic modular design for robot swarms, and have shown that they can still be used even if the low-level behaviors they combine do not return success nor failure. It is our contention that, despite their potential is not exploited in the context of the automatic modular design of robot swarms, behavior trees are a control architecture that is worth exploring. In particular, we reckon that the inherent modularity they offer could be exploited by future automatic modular design methods. In fact, behavior trees can be easily manipulated without compromising their structural integrity, which allows for the use of tailored optimization algorithms based on local manipulations.

We devised Maple to be as similar as possible to Chocolate: the two methods share the same optimization algorithm, the same set of predefined modules, and generate control software on the basis of the same reference model. The only difference between Maple and Chocolate is the control architecture adopted. We conducted three studies based on two missions: FORAGING and AGGREGATION to assess the implications of adopting behavior trees as a control architecture. In the first study, we assessed Maple's ability to cross the reality gap satisfactorily by comparing its performance in simulation and in reality against Chocolate and EvoStick, an evolutionary swarm robotics method. In the second study, we investigated the effect of the design budget on Maple and Chocolate. In the third study, we explored different variants of Maple's control architecture.

Our main findings are the following. (A) The results show that Maple is robust to the reality gap. Indeed, Maple and Chocolate performed similarly, and they suffered from a reduced performance drop with respect to EvoStick. These results confirm Francesca et al. (2014) conjecture that AutoMoDe is robust to the reality gap due to its modular nature. They also indicate that the architecture into which the predefined modules are combined is a secondary issue. (B) The study on the effect of the design budget has shown that: (i) the restrictions on the structure of Maple's behavior trees inhibit its expressiveness, indeed, for FORAGING, Maple is unable to express some efficient solution that Chocolate could generate;

\footnotetext{
${ }^{2}$ The authors also adapted their system for the onboard evolution of a swarm of nine Xpucks (Jones et al., 2019)

${ }^{3}$ Neupane and Goodrich (2019) proposed a method based on the grammatical evolution of behavior trees for robot swarms, but their experiments were conducted in simulation only and the focus of the paper is mainly ported on the evolutionary approach rather than on behavior trees.
} 
(ii) Maple converges to efficient solutions faster than Chocolate because of the smaller search space. The restrictions of Maple, imposed by the absence of natural termination criteria in the low-level behaviors adopted, appear to be a double-edged sword: they facilitate the initial search for efficient solutions, but curb the expressiveness of behavior trees. (C) When adopting the low-level behaviors of Chocolate, none of the variants considered outperformed Maple in both missions. Overall, our three studies indicate that behavior trees can be used in the particular context of swarm robotics in which low-level behaviors typically do not have a natural termination criterion. However, they also suggest that behavior trees only offer a benefit over probabilistic finite state machines when the design budget is small.

Future work could develop along two avenues. The first one could be dedicated to further investigate the use of Vanilla's and Chocolate's low-level behaviors as action nodes of behavior trees. For example, the control software generated by Maple with different design budgets could be assessed in robot experiments. The same holds for control software generated by Maple's variants. Also, further variants could be explored by relaxing the restrictions on the number of levels, branches, and leaves. For the relevant ones, the effect of the design budget could be investigated. As a second avenue, future work could be devoted to developing an ad-hoc optimization algorithm that takes advantage of the inherent modularity of behavior trees. Local search algorithms, such as iterative improvement and simulated annealing, have shown to be promising algorithms for the automatic modular design of swarm behaviors (Kuckling et al., 2019,2020 ) and could serve as starting points.

\section{REFERENCES}

Bagnell, J. A., Cavalcanti, F., Cui, L., Galluzzo, T., Hebert, M., Kazemi, M., Klingensmith, M., Libby, J., Liu, T. Y., Pollard, N., Pivtoraiko, M., Valois, J.-S., and Zhu, R. (2012). An integrated system for autonomous robotics manipulation. In IEEE/RSJ International Conference on Intelligent Robots and Systems, IROS, pages 2955-2962, Piscataway, NJ, USA. IEEE.

Balaprakash, P., Birattari, M., and Stützle, T. (2007). Improvement strategies for the F-Race algorithm: sampling design and iterative refinement. In Bartz-Beielstein, T., Blesa, M. J., Blum, C., Naujoks, B., Roli, A., Rudolph, G., and Sampels, M., editors, Hybrid Metaheuristics, 4th International Workshop, HM 2007, volume 4771 of LNCS, pages 108-122, Berlin, Germany. Springer.

Beni, G. (2004). From swarm intelligence to swarm robotics. In Şahin, E. and Spears, W. M., editors, Swarm Robotics, $S A B$, volume 3342 of $L N C S$, pages 1-9, Berlin, Germany. Springer.

Birattari, M., Ligot, A., Bozhinoski, D., Brambilla, M., Francesca, G., Garattoni, L., Garzón Ramos, D., Hasselmann, K., Kegeleirs, M., Kuckling, J., Pagnozzi, F., Roli, A., Salman, M., and Stützle, T. (2019). Automatic off-line design of robot swarms: a manifesto. Frontiers in Robotics and AI, 6:59.

Birattari, M., Ligot, A., and Hasselmann, K. (2020). Disentangling automatic and semi-automatic approaches to the optimization-based design of control software for robot swarms. Nature Machine Intelligence, 2(9):494-499.

Bozhinoski, D. and Birattari, M. (2018). Designing control software for robot swarms: Software engineering for the development of automatic design methods. In Proceedings of the 1st International Workshop on Robotics Software Engineering, RoSE, pages 33-35, New York, NY, USA. ACM.

Brambilla, M., Ferrante, E., Birattari, M., and Dorigo, M. (2013). Swarm robotics: a review from the swarm engineering perspective. Swarm Intelligence, 7(1):1-41.

Brooks, R. A. (1986). A robust layered control system for a mobile robot. IEEE Journal on Robotics and Automation, 2(1):14-23.

Brooks, R. A. (1991). Intelligence without representation. Artificial Intelligence, 47:139-159.

Brooks, R. A. (1992). Artificial life and real robots. In Varela, F. J. and Bourgine, P., editors, Towards a Practice of Autonomous Systems. Proceedings of the First European Conference on Artificial Life, pages 3-10, Cambridge, MA, USA. MIT Press.

Bäck, T., Fogel, D. B., and Michalewicz, Z., editors (1997). Handbook of Evolutionary Computation. IOP Publishing Ltd., Bristol, UK, first edition.

Champandard, A. J. (2007). Understanding behavior trees. http://aigamedev.com/open/articles/ bt-overview/.

Champandard, A. J., Dawe, M., and Hernandez-Cerpa, D. (2010). Behavior trees: Three ways of cultivating game AI. https://www.gdcvault.com/play/1012744/Behavior-Trees-Three-Ways-of. Game Developers Conference, AI Summit.

Christensen, A. L. and Dorigo, M. (2006). Evolving an integrated phototaxis and hole-avoidance behavior 
for a swarm-bot. In Rocha, L. M., Yaeger, L. S., Bedau, M. A., Floreano, D., Goldstone, R. L., and Vespignani, A., editors, Artificial Life X: Proceedins of the Tenth International Conference on the Simulation and Synthesis of Living Systems, pages 248-254, Cambridge, MA, USA. MIT Press. A Bradford Book.

Colledanchise, M. and Ögren, P. (2018). Behavior Trees in Robotics and AI: An Introduction. Chapman $\&$ Hall/CRC Artificial Intelligence and Robotics Series. CRC Press, Boca Raton, FL, 1st edition.

Conover, W. J. (1999). Practical Nonparametric Statistics. Wiley series in probability and statistics, applied probability and statistics section. John Wiley \& Sons, New York, NY, USA, third edition.

Şahin, E. (2004). Swarm robotics: from sources of inspiration to domains of application. In Şahin, E. and Spears, W. M., editors, Swarm Robotics, SAB, volume 3342 of LNCS, pages 10-20, Berlin, Germany. Springer.

Dorigo, M., Birattari, M., and Brambilla, M. (2014). Swarm robotics. Scholarpedia, 9(1):1463.

Floreano, D., Husbands, P., and Nolfi, S. (2008). Evolutionary robotics. In Siciliano, B. and Khatib, O., editors, Springer Handbook of Robotics, Springer Handbooks, pages 1423-1451. Springer, Berlin, Heidelberg, Germany. First edition.

Francesca, G. and Birattari, M. (2016). Automatic design of robot swarms: achievements and challenges. Frontiers in Robotics and AI, 3(29):1-9.

Francesca, G., Brambilla, M., Brutschy, A., Garattoni, L., Miletitch, R., Podevijn, G., Reina, A., Soleymani, T., Salvaro, M., Pinciroli, C., Mascia, F., Trianni, V., and Birattari, M. (2015). AutoMoDeChocolate: automatic design of control software for robot swarms. Swarm Intelligence, 9(2-3):125-152.

Francesca, G., Brambilla, M., Brutschy, A., Trianni, V., and Birattari, M. (2014). AutoMoDe: a novel approach to the automatic design of control software for robot swarms. Swarm Intelligence, 8(2):89112.

Friedman, M. (1937). The use of ranks to avoid the assumption of normality implicit in the analysis of variance. Journal of the American Statistical Association, 32(200):675-701.

Friedman, M. (1939). A correction: The use of ranks to avoid the assumption of normality implicit in the analysis of variance. Journal of the American Statistical Association, 34(205):109.

Garattoni, L. and Birattari, M. (2016). Swarm robotics. In Webster, J. G., editor, Wiley Encyclopedia of Electrical and Electronics Engineering, pages 1-19. John Wiley \& Sons, Hoboken, NJ, USA.

Garattoni, L., Francesca, G., Brutschy, A., Pinciroli, C., and Birattari, M. (2015). Software infrastructure for e-puck (and TAM). Technical Report TR/IRIDIA/2015-004, IRIDIA, Université libre de Bruxelles, Belgium.

Garzón Ramos, D. and Birattari, M. (2020). Automatic design of collective behaviors for robots that can display and perceive colors. Applied Sciences, 10(13):4654.

Geman, S., Bienenstock, E., and Doursat, R. (1992). Neural networks and the bias/variance dilemma. Neural Computation, 4(1):1-58.

Gutiérrez, Á., Campo, A., Dorigo, M., Donate, J., Monasterio-Huelin, F., and Magdalena, L. (2009). Open e-puck range \& bearing miniaturized board for local communication in swarm robotics. In Kosuge, K., editor, IEEE International Conference on Robotics and Automation, ICRA, pages 3111-3116, Piscataway, NJ, USA. IEEE.

Hasselmann, K., Ligot, A., Francesca, G., and Birattari, M. (2018a). Reference models for AutoMoDe. Technical Report TR/IRIDIA/2018-002, IRIDIA, Université libre de Bruxelles, Belgium.

Hasselmann, K., Robert, F., and Birattari, M. (2018b). Automatic design of communication-based behaviors for robot swarms. In Dorigo, M., Birattari, M., Garnier, S., Hamann, H., Montes de Oca, M., Solnon, C., and Stützle, T., editors, Swarm Intelligence - ANTS, volume 11172 of LNCS, pages 16-29, Cham, Switzerland. Springer.

Hauert, S., Zufferey, J.-C., and Floreano, D. (2009). Evolved swarming without positioning information: an application in aerial communication relay. Autonomous Robots, 26(1):21-32.

Hu, D., Gong, Y., Hannaford, B., and Seibel, E. J. (2015). Semi-autonomous simulated brain tumor ablation with RavenII surgical robot using behavior tree. In IEEE International Conference on Robotics and Automation, ICRA, pages 3868-3875, Piscataway, NJ, USA. IEEE.

Isla, D. (2005). Handling complexity in the Halo 2 AI. In Game Developers Conference, volume 12.

Jakobi, N., Husbands, P., and Harvey, I. (1995). Noise and the reality gap: the use of simulation in evolutionary robotics. In Morán, F., Moreno, A., Merelo, J. J., and Chacón, P., editors, Advances in Artificial Life: Third european conference on artificial life, volume 929 of Lecture Notes in Artificial 
Intelligence, pages 704-720, Berlin, Germany. Springer.

Jones, S., Studley, M., Hauert, S., and Winfield, A. (2018). Evolving behaviour trees for swarm robotics. In Groß, R., Kolling, A., Berman, S., Frazzoli, E., Martinoli, A., Matsuno, F., and Gauci, M., editors, Distributed Autonomous Robotic Systems (DARS), volume 6 of SPAR, pages 487-501, Cham, Switzerland. Springer.

Jones, S., Winfield, A., Hauert, S., and Studley, M. (2019). Onboard evolution of understandable swarm behaviors. Advanced Intelligent Systems, 1(6):1900031.

Kuckling, J., Ligot, A., Bozhinoski, D., and Birattari, M. (2018a). Behavior trees as a control architecture in the automatic modular design of robot swarms. In Dorigo, M., Birattari, M., Blum, C., Christensen, A. L., Reina, A., and Trianni, V., editors, Swarm Intelligence - ANTS, volume 11172 of LNCS, pages 30-43, Cham, Switzerland. Springer.

Kuckling, J., Ligot, A., Bozhinoski, D., and Birattari, M. (2018b). Search space for AutoMoDe-Chocolate and AutoMoDe-Maple. Technical Report TR/IRIDIA/2018-012, IRIDIA, Université Libre de Bruxelles, Brussels, Belgium.

Kuckling, J., Stützle, T., and Birattari, M. (2020). Iterative improvement in the automatic modular design of robot swarms. PeerJ Computer Science. In press.

Kuckling, J., Ubeda Arriaza, K., and Birattari, M. (2019). Simulated annealing as an optimization algorithm in the automatic modular design of robot swarms. In Beuls, K., Bogaerts, B., Bontempi, G., Geurts, P., Harley, N., Lebichot, B., Lenaerts, T., Gilles, L., and Van Eecke, P., editors, Proceedings of the Reference AI \& ML Conference for Belgium, Netherlands \& Luxemburg, BNAIC/BENELEARN 2019, volume 2491 of CEUR Workshop Proceedings, Aachen, Germany. CEUR-WS.org.

Ligot, A. and Birattari, M. (2018). On mimicking the effects of the reality gap with simulation-only experiments. In Dorigo, M., Birattari, M., Garnier, S., Hamann, H., Montes de Oca, M., Solnon, C., and Stützle, T., editors, Swarm Intelligence - ANTS, volume 11172 of LNCS, pages 109-122, Cham, Switzerland. Springer.

Ligot, A. and Birattari, M. (2019). Simulation-only experiments to mimic the effects of the reality gap in the automatic design of robot swarms. Swarm Intelligence, pages 1-24.

Ligot, A., Kuckling, J., Bozhinoski, D., and Birattari, M. (2020). Automatic modular design of robot swarms using behavior trees as a control architecture: Supplementary material. http://iridia.ulb.ac. be/supp/IridiaSupp2020-009/index.html.

Lim, C.-U., Baumgarten, R., and Colton, S. (2010). Evolving behaviour trees for the commercial game DEFCON. In Di Chio, C., Cagnoni, S., Cotta, C., Ebner, M., Ekárt, A., Esparcia-Alcázar, A. I., Goh, C.-K., Merelo, J. J., Neri, F., Preuss, M., Togelius, J., and Yannakakis, G. N., editors, Applications of Evolutionary Computation, volume 6024 of LNCS, pages 100-110, Berlin, Germany. Springer.

Lipson, H. (2005). Evolutionary robotics and open-ended design automation. In Biomimetics: Biologically Inspired Technologies, volume 17, pages 129-155. CRC Press, Boca Raton, FL.

López-Ibáñez, M., Dubois-Lacoste, J., Pérez Cáceres, L., Birattari, M., and Stützle, T. (2016). The irace package: Iterated racing for automatic algorithm configuration. Operations Research Perspectives, 3:43-58.

Marzinotto, A., Colledanchise, M., Smith, C., and Ögren, P. (2014). Towards a unified behavior trees framework for robot control. In IEEE International Conference on Robotics and Automation, ICRA, pages 5420-5427, Piscataway, NJ, USA. IEEE.

Mondada, F., Bonani, M., Raemy, X., Pugh, J., Cianci, C., Klaptocz, A., Magnenat, S., Zufferey, J.-C., Floreano, D., and Martinoli, A. (2009). The e-puck, a robot designed for education in engineering. In Gonçalves, P., Torres, P., and Alves, C., editors, Proceedings of the 9th Conference on Autonomous Robot Systems and Competitions, pages 59-65, Castelo Branco, Portugal. Instituto Politécnico de Castelo Branco.

Nehaniv, C. L. and Dautenhahn, K. (2002). Imitation in animals and artifacts. MIT press, first edition.

Neupane, A. and Goodrich, M. (2019). Learning swarm behaviors using grammatical evolution and behavior trees. In Kraus, S., editor, Twenty-Eighth International Joint Conference on Artificial Intelligence (IJCAI-19), pages 513-520. IJCAI.

Paxton, C., Hundt, A., Jonathan, F., Guerin, K., and Hager, G. D. (2017). CoSTAR: Instructing collaborative robots with behavior trees and vision. In IEEE International Conference on Robotics and Automation, ICRA, pages 564-571, Piscataway, NJ, USA. IEEE.

Perez, D., Nicolau, M., O'Neill, M., and Brabazon, A. (2011). Evolving behaviour trees for the Mario AI 
competition using grammatical evolution. In Di Chio, C., Cagnoni, S., Cotta, C., Ebner, M., Ekárt, A., Esparcia-Alcázar, A. I., Merelo, J. J., Neri, F., Preuss, M., Richter, H., Togelius, J., and Yannakakis, G. N., editors, Applications of Evolutionary Computation, volume 6624 of Lecture Notes in Computer Science, pages 123-132, Berlin, Germany. Springer.

Pinciroli, C., Trianni, V., O’Grady, R., Pini, G., Brutschy, A., Brambilla, M., Mathews, N., Ferrante, E., Di Caro, G. A., Ducatelle, F., Birattari, M., Gambardella, L. M., and Dorigo, M. (2012). ARGoS: a modular, parallel, multi-engine simulator for multi-robot systems. Swarm Intelligence, 6(4):271-295.

Quinn, M., Smith, L., Mayley, G., and Husbands, P. (2003). Evolving controllers for a homogeneous system of physical robots: structured cooperation with minimal sensors. Philosophical Transactions of the Royal Society of London. Series A: Mathematical, Physical and Engineering Sciences, 361(1811):2321-2343.

Salman, M., Ligot, A., and Birattari, M. (2019). Concurrent design of control software and configuration of hardware for robot swarms under economic constraints. PeerJ Computer Science, 5:e221.

Silva, F., Duarte, M., Correia, L., Oliveira, S. M., and Christensen, A. L. (2016). Open issues in evolutionary robotics. Evolutionary Computation, 24(2):205-236.

Spaey, G., Kegeleirs, M., Garzón Ramos, D., and Birattari, M. (2019). Comparison of different exploration schemes in the automatic modular design of robot swarms. In Beuls, K., Bogaerts, B., Bontempi, G., Geurts, P., Harley, N., Lebichot, B., Lenaerts, T., Gilles, L., and Van Eecke, P., editors, Proceedings of the Reference AI \& ML Conference for Belgium, Netherlands \& Luxemburg, BNAIC/BENELEARN 2019, volume 2491 of CEUR Workshop Proceedings, Aachen, Germany. CEUR-WS.org.

Spears, W. M., Spears, D., Hamann, J. C., and Heil, R. (2004). Distributed, physics-based control of swarms of vehicles. Autonomous Robots, 17(2):137-162.

Stranieri, A., Turgut, A. E., Salvaro, M., Garattoni, L., Francesca, G., Reina, A., Dorigo, M., and Birattari, M. (2013). IRIDIA's arena tracking system. Technical Report TR/IRIDIA/2013-013, IRIDIA, Université libre de Bruxelles, Belgium.

Trianni, V. (2008). Evolutionary Swarm Robotics. Springer, Berlin, Germany.

Trianni, V. (2014). Evolutionary robotics: model or design? Frontiers in Robotics and AI, 1:13.

Trianni, V. and Nolfi, S. (2009). Self-organizing sync in a robotic swarm: a dynamical system view. IEEE Transactions on Evolutionary Computation, 13(4):722-741.

Ögren, P. (2012). Increasing modularity of UAV control systems using computer game behavior trees. In Thienel, J. et al., editors, AIAA guidance, navigation, and control conference 2012, pages 358-393. AIAA Meeting Papers. 